\title{
THE ROLE OF SUPERCONDUCTIVITY AND CRYOGENICS IN THE NEUTRINO FACTORY
}

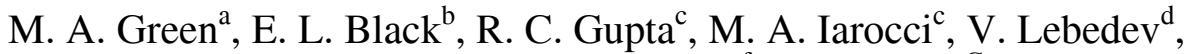 \\ J. R. Miller ${ }^{\mathrm{e}}$, R. B. Palmer ${ }^{\mathrm{c}}$, H. S. Padamsee ${ }^{\mathrm{f}}$, B. L Parker ${ }^{\mathrm{C}}$, S. Prestemon ${ }^{\mathrm{a}}$, \\ and R. J. Weggel ${ }^{\mathrm{c}}$ \\ a. Lawrence Berkeley National Laboratory, Berkeley CA 94720, USA \\ b. Fermi National Laboratory, Batavia IL 60510, USA \\ c. Brookhaven National Laboratory, Upton NY 11973, USA \\ d. Jefferson Lab, Newport News VA 23606, USA \\ e. National High Magnetic Field Laboratory, Tallahassee FL 32310, USA \\ f. Cornell University, Ithica NY 14853, USA
}

\begin{abstract}
The proposed neutrino factory will produce a defined beam of neutrinos from the decay of muons in a storage ring[1,2,3]. The storage ring will be oriented so that the neutrinos can be detected at one or more detectors several thousand kilometers from the storage ring. This report presents an overview of the proposed neutrino factory and its subsystems that use cryogenics. Superconducting magnets will be used in the following ways in the neutrino factory; 1) the outsert solenoid for the $20 \mathrm{~T}$ pion capture system, 2) the decay channel where pions decay to muons, 3) the muon phase rotation system, 4) the muon cooling system, 5) focusing during the first stage of muon acceleration, 6) bending and focusing magnets in the re-circulating linac accelerator and 7) bending and focusing magnets in the muon storage ring where the neutrino beams are generated. Low temperature superconducting RF cavities will be used to accelerate the muons from about $200 \mathrm{MeV}$ to $20 \mathrm{GeV}$. The muon cooling system uses liquid hydrogen absorbers at $20 \mathrm{~K}$ to reduce the emittance of the muon beam before it is accelerated to full energy.
\end{abstract}

\section{THE PION CAPTURE SOLENOID}

The capture solenoid is a hybrid magnet that develops an induction of $20 \mathrm{~T}$ around a mercury jet target[4]. The clear bore of this solenoid is $150 \mathrm{~mm}$. The solenoid can capture pions with a transverse momentum up to $225 \mathrm{MeV} / \mathrm{c}$. The hybrid solenoid consists of three water- cooled copper coils that are within a large cable in conduit superconducting (CICC) solenoid made from a niobium tin conductor. At one end of the water-cooled copper coils is a plug of cobalt-iron what enhances the magnetic induction at the target and makes the field more uniform in that region. Figure 1 shows the location of the pole with respect to the water-cooled copper coils and the superconducting coils surrounding the conventional 
insert coil system. The three superconducting coils that are closest to the target will be fabricated from a niobium-tin CICC conductor. The two-solenoid sections downstream from the niobium-tin CICC are also CICC coils made using niobium titanium. The capture solenoid is characterized by very high radiation heating. The solenoid are lined with a water-cooled tungsten copper liner that absorbs the radiation heat load. The position of the superconducting coils is dictated by radiation heating and radiation damage to the insulation.

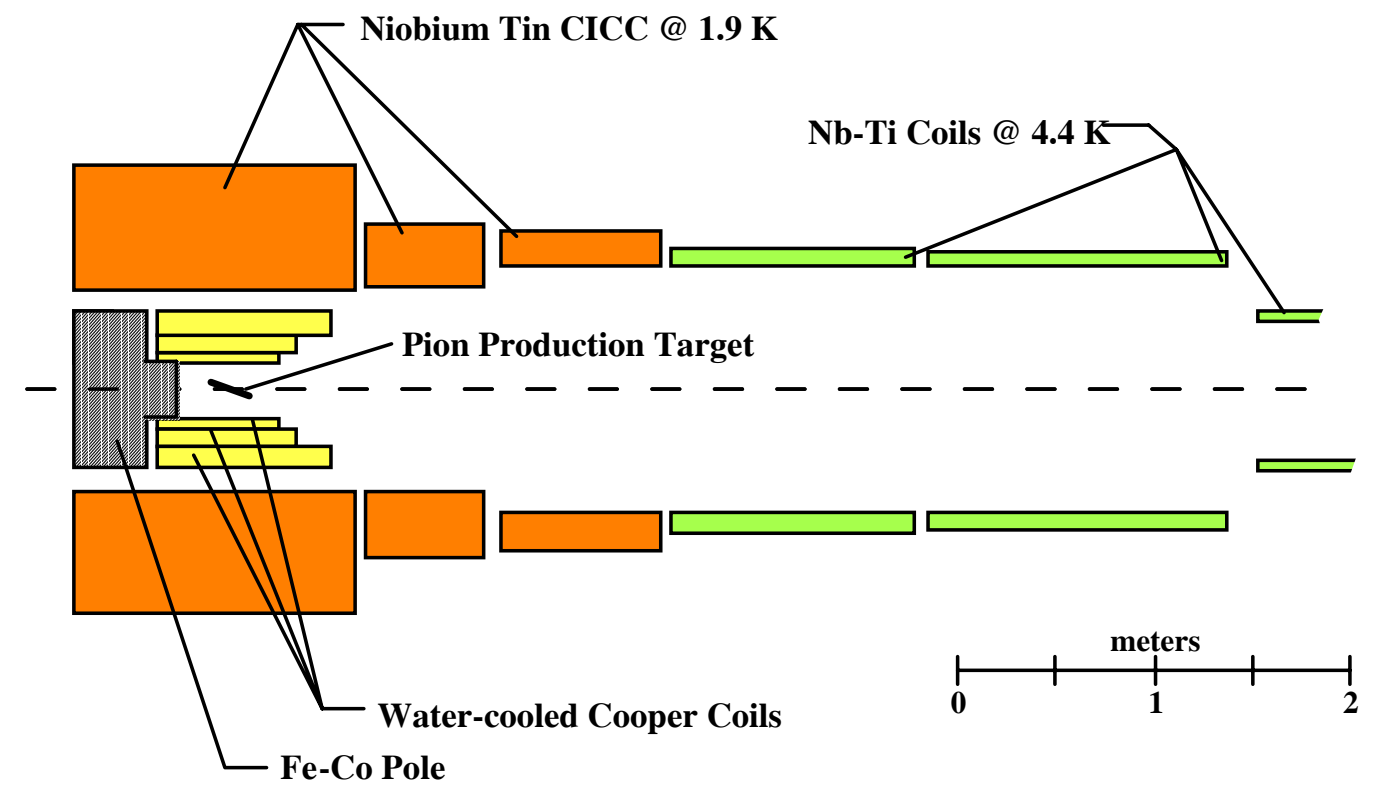

FIGURE 1. A Schematic Representation of the Hybrid Capture Solenoid System that is around and downstream from the Pion Production Target

The cable in conduit solenoids shown in Figure 1 have a total stored magnetic energy of $600 \mathrm{MJ}$. This stored energy is similar to the ITER coils, which have a similar method of construction. Since the niobium-tin outsert coils are expected to generate $14 \mathrm{~T}$ alone at the target, it is assumed that the coils would operate at $2.5 \mathrm{~K}$. Given the improvement in the quality of niobium-tin is the last two years, it is not clear that the temperature has to be that low. By the time the superconducting outsert coils would have to be fabricated, conductor improvements may allow the coils to operate at $4.4 \mathrm{~K}$. Recent experiments at LBNL have demonstrated that niobium-tin dipoles can generate up to $14.7 \mathrm{~T}$ with niobium-tin at 4.4 $\mathrm{K}[5]$.

All of the superconducting solenoids that are downstream from the solenoids shown in Figure 1 can be made from niobium-titanium that is potted and indirectly cooled. As one moves downstream from the target the on axis magnetic induction decay adiabatically from $20 \mathrm{~T}$ at the target to $1.25 \mathrm{~T}$ at a point 18 meter downstream from the target. The magnet clear bore diameter of the solenoid increases from $150 \mathrm{~mm}$ to $600 \mathrm{~mm}$ in proportion to one over the square root of the magnetic induction on axis.

\section{MUON DECAY AND PHASE ROTATION}

Within this decay and phase rotation region, there are four types of solenoids[6,7]. They are: 1) There is a decay section that has a warm bore diameter of $600 \mathrm{~mm}$. Around this warm bore is a water-cooled copper shielding that is 50 to $100-\mathrm{mm}$ thick. The solenoid cryostat warm bore is about $800 \mathrm{~mm}$. The 18 meters of decay solenoid is divided into six cryostats that are each 2.9-meters long. This same type of magnet can be used for 
the 9-meter long mini-cooling sections on either side of the field flip solenoid. As a result, it is assumed that there are twelve magnets of this type. 2) The first induction linac solenoids extend $110 \mathrm{~m}$ from the pion decay section to the first mini-cooler. These magnets have a beam bore diameter of $600 \mathrm{~mm}$. Around this bore is a 10-mm thick watercooled copper radiation shield. The warm bore of this magnet cryostat is $620 \mathrm{~mm}$ in diameter. There are one hundred-ten magnets of this type. 3) The second and third induction linacs and the drift between them extend $190 \mathrm{~m}$ from the second mini-cooler to the start of the matching section before the muon bunching section. These solenoids have no radiation shield and have a cryostat warm bore diameter of $600 \mathrm{~mm}$. There are one hundred-ninety magnets of this type. 4) The field flip solenoid between the two mincooling sections is 2.0 -meters long with a warm bore diameter of $400 \mathrm{~mm}$. There is only one of these magnets. 
TABLE 1. Induction Linac Phase Rotation Solenoid Parameters

$$
1 \text { st } 110 \mathrm{~m} \quad \text { Last } 190 \mathrm{~m}
$$

$\begin{array}{lcc}\text { Magnet Physical Parameters } & & \\ \text { Number of Cells of This Type } & 110 & 190 \\ \text { Cell Length (mm) } & 1000.0 & 1000.0 \\ \text { Magnet Cryostat Length (mm) } & 900.0 & 900.0 \\ \text { Magnet Coil Package Length (mm) } & 860.0 & 860.0 \\ \text { Length of Each S/C Coil (mm) } & 360.0 & 360.0 \\ \text { Inner Cryostat Radius (mm) } & 310.0 & 300.0 \\ \text { S/C Coil Inner Radius (mm) } & 334.0 & 324.0 \\ \text { S/C Coil Thickness (mm) } & 9.6 & 9.6 \\ \text { Magnet Electrical Parameters } & & \\ \text { Average Central Induction (T) } & 1.25 & 1.25 \\ \text { On Axis Induction Variation (\%) } & \pm 2.5 & \pm 2.5 \\ \text { Peak Induction in the Windings (T) } & \sim 1.6 & \sim 1.6 \\ \text { Number of Turns per Cell } & 2532 & 2532 \\ \text { Magnet Design Current (A) } & 392.8 & 392.8 \\ \text { Magnet Design Operating Temperature (K) } & 4.4 & 4.4 \\ \text { Conductor Critical Current at Operating T (A) } & \sim 1600 & \sim 1600 \\ \text { Magnet Stored Energy per Cell E (kJ) } & 224 & 211 \\ \text { Magnet Self Inductance per Cell (H) } & 2.90 & 2.74\end{array}$

Table 1 above shows the design parameters for the induction linac phase-rotation solenoids. The larger bore decay channel solenoids, the mini cooling solenoids and 2meter long field flip solenoid is not included in Table 1. Figure 2 below shows a crosssection of a typical induction cell downstream from the mini-cooler section. Figure 2 shows the cell superconducting solenoid, and the induction linac cores. The superconducting magnets in the phase-rotation and mini-cooling section are described in Reference . 


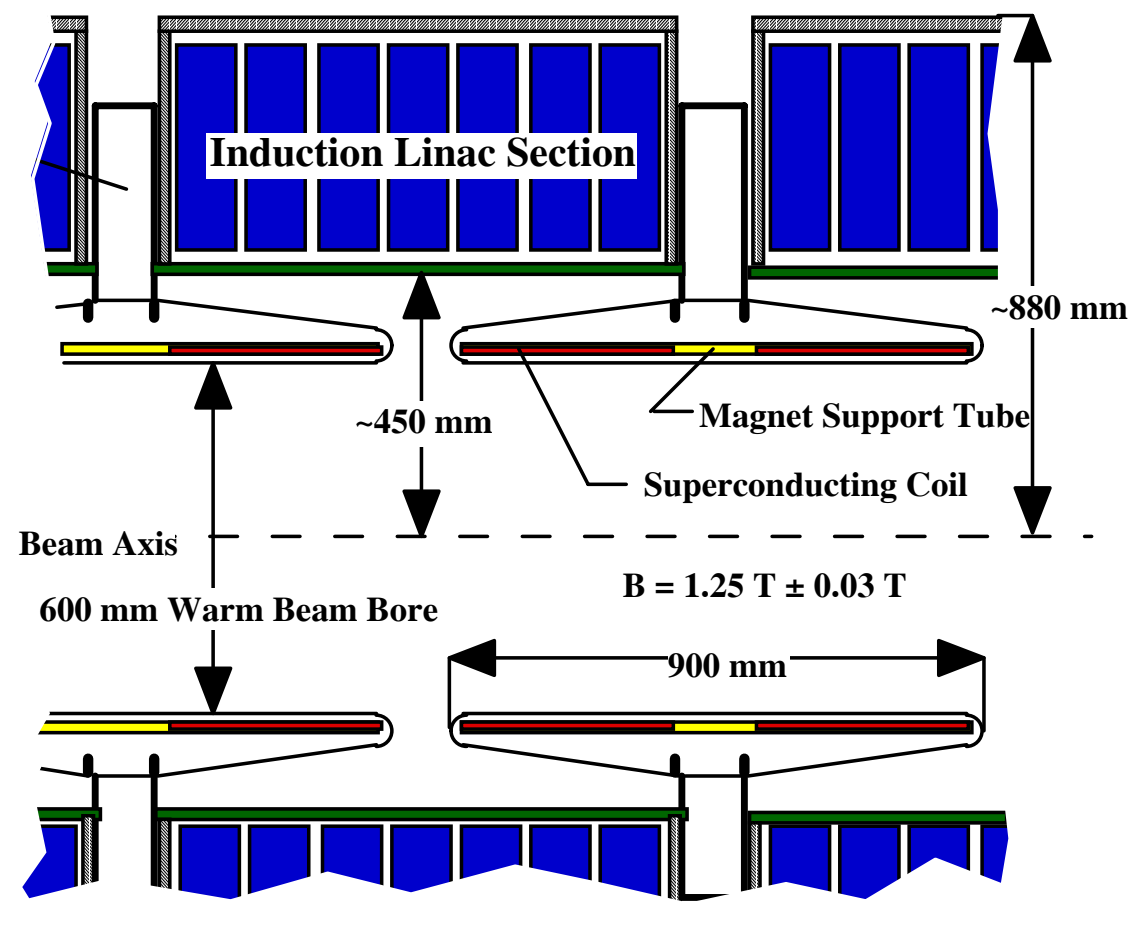

FIGURE 2. A Cross-section of the Solenoid in an Induction Cell downstream from the Mini-cooler SOLENOIDS AND ABSORBERS FOR THE COOLING CHANNEL

The beam bunching and cooling section is between phase-rotation section and the linear accelerator section[8,9]. The bunching section starts with a matching section that is 11-meters long. This section matches the phase-rotation induction of $1.25 \mathrm{~T}$ with the field flipping structure that characterizes the bunching and cooling sections. Downstream from the matching section are twenty cells of beam bunching. Each cell is $2.75 \mathrm{~m}$ long and each cell contains four 201.25 MHz RF cavities and one 402.5 MHz RF cavity. The beambunching section is followed by seventeen cells of muon cooling that are $2.75-\mathrm{m}$ long. These cells have four $201.25 \mathrm{MHz}$ RF cavities and a hydrogen absorber that is in the bore of a field flip solenoid. The final cooling section consists of thirty-seven cells that are 1.65-meters long. The 1.65-meter long cooling cells contains two $210.25 \mathrm{MHz}$ RF cavities and a short hydrogen absorber that is in a field flip solenoid. The final matching section is between the short cell cooling section and the linear accelerator. The total length of the bunching and cooling section is a bit over 184 meters. The first $70 \mathrm{MeV}$ of muon acceleration occurs in the matching section between the last cooling section and the linear accelerator section.

The matching section consists of four 2.75-meter long cells and about 3 meters of solenoid (part of the phase rotation section) that have a warm bore diameter of $600 \mathrm{~mm}$. These solenoids must be designed to withstand longitudinal forces of up to 75 metric tons that are imparted on them by the matching process. The solenoids in the four matching section cells are the same as the $2.75-\mathrm{m}$ long cells in the beam bunching section downstream. The twenty 2.75 -meter long bunching cells are the same as the 2.75 -meter long cooling cells. The warm bore aperture of the of the A coils for a 2.75-meter long cooling cell must be about $650 \mathrm{~mm}$ in order to accommodate a liquid hydrogen absorber. The warm bore aperture for the beam bunching cell flux reversal coils must be the same in 
order to accommodate a $402.5 \mathrm{MHz}$ RF cavity. Room temperature service ports to the 402.5 MHz RF cavity can go out through the flux reversal magnet cryostat between the flux reversal coils. (A coils in Figure 3.) Table 2 below shows the number of cells of each type, the minimum aperture requirements for the magnets and the maximum coil current densities for the coils in each cell type. Because the bunching and cooling cell solenoids are constantly changing polarity, there is almost no stray field from these solenoids at $\mathrm{R}=$ 10 meters.

Magnet parameters and a magnet cross section for the 2.75-meter long bunching and cooling cell magnets are shown in Table 2 and Figure 3. The solenoids in the 2.75-meter long cells are the same for both bunching and cooling cells. Parameters for the 1.65-meter long cooling cell magnets are also shown in Table 2. The typical 1.65-meter long cell shown in Table 2 is used for both the cooling section and the matching between the cooling section and the linear accelerator section. More information on the 1.65-meter long cell superconducting solenoids can be found in Reference 9.

TABLE 2. Superconducting Magnet Parameters for the Beam Bunching Cells and the Muon Cooling Cells

\section{Parameter}

Number of Cells of This Type

Cell Length (mm)

Maximum Space for the RF Cavity (mm)

Number of 201.25 MHz RF Cavities per Cell

Number of 402,5 MHz RF Cavities per Bunching Cell

A Magnet Cryostat Length (mm)

B Magnet Cryostat Length ( $\mathrm{mm})$

Aperture for the A Magnet $(\mathrm{mm})$

Aperture for the B Magnet (mm)

Maximum A Coil Current Density $\left(\mathrm{A} \mathrm{mm}^{-2}\right)$

Maximum B Coil Current Density $\left(\mathrm{A} \mathrm{mm}^{-2}\right.$ )

Maximum Cell Stored Energy (MJ)

Maximum Longitudinal Warm to Cold Force (MN)

Number of Longitudinal Supports per Coil

The Force Pushing the Flux Reversal Coils Apart (MN)

\subsection{5-m Cell 1.65-m Cell}

$\begin{array}{cc}41 & 44 \\ 2750 & 1650 \\ 1966 & 1108 \\ 4 & 2 \\ 1 & \text { NA } \\ 784 & 542 \\ 283 & 209 \\ 650 & 370 \\ 1390 & 1334 \\ 128.04 & 99.81 \\ 98.83 & 105.53 \\ 13.2 & 17.6 \\ 0.74 & 1.20 \\ 4 & 6 \text { to } 8 \\ 3.22 & 19.40\end{array}$




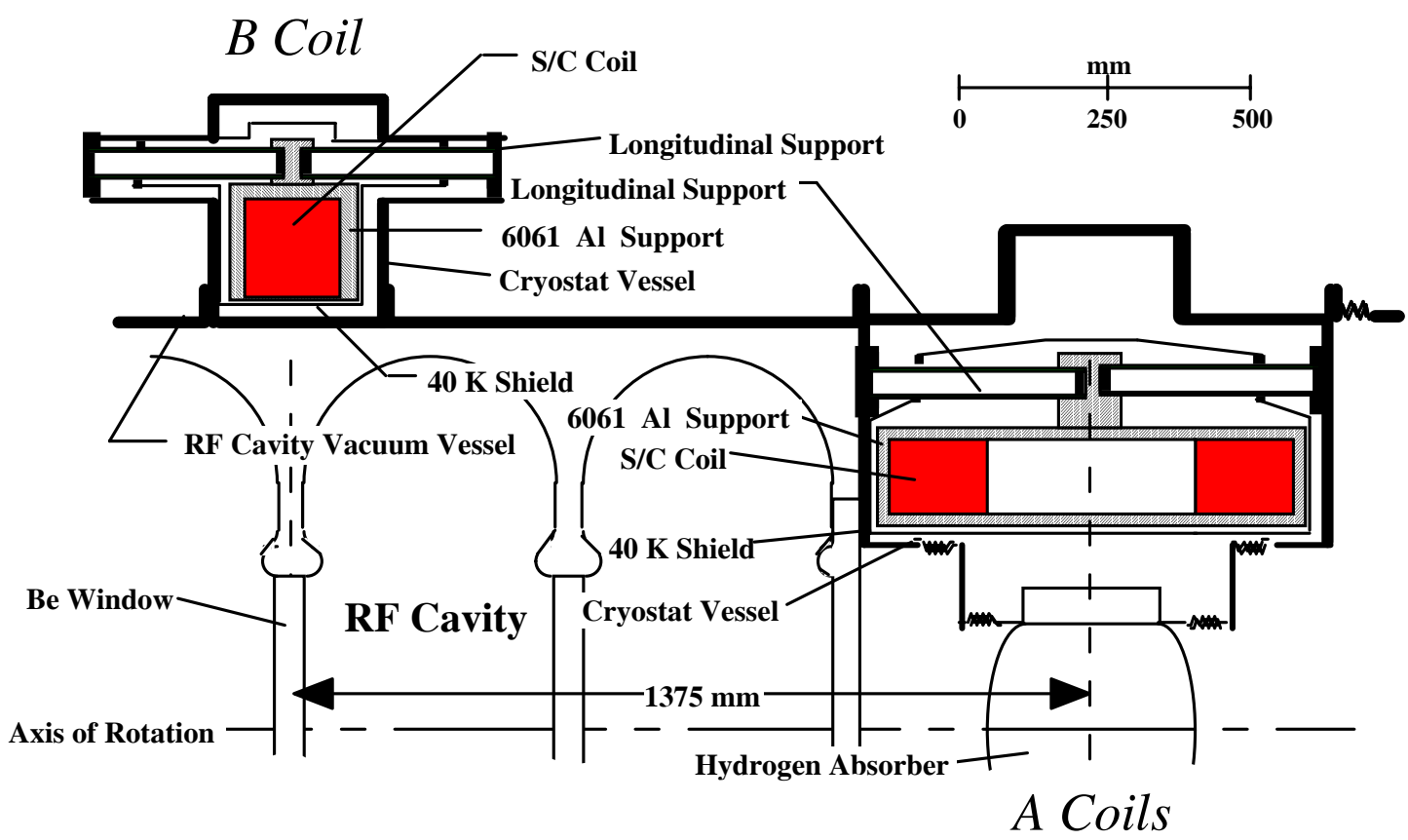

FIGURE 3. A Quarter Section for a Typical 2.75-meter Long Cooling Cell with a Liquid Hydrogen Absorber and a Four Cell 201.25 MHz RF Cavity with Beryllium Windows between Cavity Cells

Figure 3 shows the RF cavity and the liquid hydrogen absorber within the 2.75-meter long cooling cell. The RF cavity sits in vacuum. This vacuum is separated from the cryostat vacuum and the vacuum within the RF cavity. Figure 3 shows the longitudinal cold mass supports for the coils. These supports must carry 80 metric tons in the cell shown.

The hydrogen absorber and the superconducting magnets use a common refrigeration source. The absorber shown in Figure 3 must be provided with $330 \mathrm{~W}$ of cooling at $16 \mathrm{~K}$. The absorber in the 1.65 -meter long cooling cell requires $150 \mathrm{~W}$ of cooling at $16 \mathrm{~K}$. The cooling circuit for the absorbers can be used to cool the shield, intercepts and gas cooled leads for the superconducting magnets.

The highest field point for the magnets in either of the cooling cells occurs in the flux reversal coil (the A-coils shown in Figure 3). The value of highest induction in the 2.75meter long cell A-coils is about 7.4 T. The highest induction value in the 1.65 -meter long cell A-coils is about $8.5 \mathrm{~T}$. It is clear that the A-coils in the 1.65-meter long cell must operate at reduced temperature (say $2.5 \mathrm{~K}$ ) or they must be made from a niobium tin. The choice of conductor in the coils will be dictated by economics. A change in the cooling lattice could also result in a smaller peak field in the flux reversal coils.

\section{MAGNETS AND RF CAVITIES FOR THE LINEAR ACCELERATOR}

The linear pre-accelerator that accelerates the muons from $260 \mathrm{MeV}$ to $2350 \mathrm{MeV}$ is about 400-meters long.. The Linear accelerator uses superconducting RF cavities to accelerate the beam[3,10]. Between the cavities superconducting solenoids that provide focusing. Muon acceleration from $190 \mathrm{MeV}$ to $260 \mathrm{MeV}$ is provided by the matching section at the end of the muon-cooling channel. The linear accelerator is divided into three sections. The first section has 4 short modules that are 5-meters long. The second acceleration section has 16 medium length modules that are 8-meters long. The focusing solenoids for the short and medium length modules produce an induction of $2.1 \mathrm{~T}$ over a length of 1-meter. The third acceleration section has 19 long modules that are 13-meters 
long. The focusing solenoid for the long module generates $4.2 \mathrm{~T}$ over a length of 1.5meters. The three types of acceleration modules are illustrated in Figure 4. 


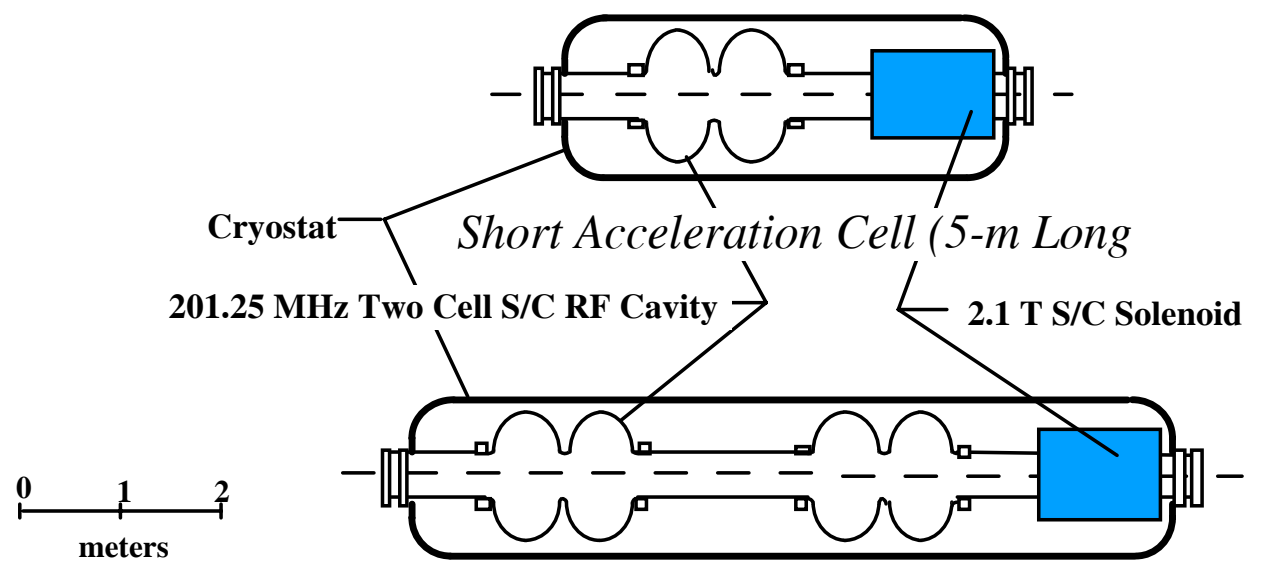

Intermediate Acceleration Cell (8-m Long)

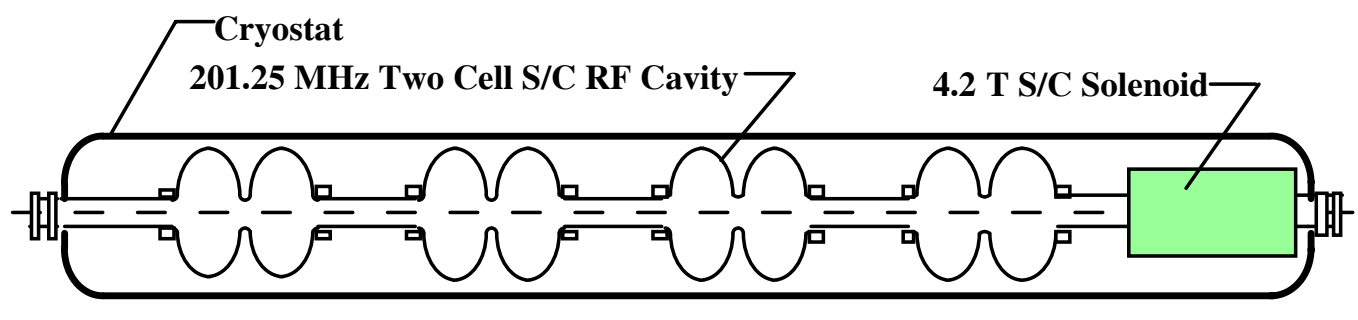

Long Acceleration Cell (13-m Long)

FIGURE 4. A Schematic of the Three Superconducting Acceleration Modules used in the Pre-accelerator.

Acceleration of the muon beam is done by $201.25 \mathrm{MHz}$ superconducting RF cavities that are capable of accelerating the muons $15 \mathrm{MV}$ per meter. The cavities are about 1.2 meters in diameter and 1.5 meters long. Each cavity consists of two coupled cells. The cavities are separated by a minimum distance of 1 meter to prevent the cavities from being closely coupled to each other. Niobium cavities cooled to $2.5 \mathrm{~K}$ were assumed for the acceleration sections.

An important issue for the superconducting RF cavities is stray field from the focusing elements. Focusing solenoids produce a stray field that reaches far from the magnet. Superconducting RF cavities are sensitive to magnetic fields at the $1 \mathrm{G}\left(10^{-4} \mathrm{~T}\right)$ level. The methods one can use to eliminate the stray field in the RF cavities are:1) The focusing solenoid should produce zero net magnetic moment. The magnetic moment due to the focusing solenoid is bucked by the moment of a larger solenoid. 2) The field from the bucking coils should be distributed in the same way as the focusing solenoid field. This suggests that the bucking solenoid surrounds the focusing solenoid so that the focusing solenoid flux returns between the two solenoids. 3) The solenoid pair can be surrounded by iron except where the muon beam passes through the solenoid. 4) An iron flux shield can be installed between the focusing solenoid magnet package and the RF cavity cells. 5) The superconducting RF cells nearest the focusing solenoid can be covered with a type 2 superconducting shield. This shield will not shield out earth's magnetic field, but it will shield out any remaining stray flux from a nearby solenoid.

Table 3 presents the mechanical and electrical parameters for the short, intermediate and long module focusing solenoids. The short and intermediate acceleration module focusing solenoids have the same length and the same design field. The focusing solenoid and its bucking coil will be powered in series with a single set of HTS and gas-cooled electrical leads. An actively shielded focusing solenoid will use about 40 percent more superconductor than one that is not actively shielded. 
The conductor for the magnets shown in Table 3 above is assumed to be a standard niobium-titanium superconductor used for MRI magnets. For the purposes of the study, the cross-sectional area of the conductor is $1.6 \mathrm{~mm}^{2}$ and the copper to superconductor ratio is 4 . Since the coils are mounted on a conducting bore tube, the magnets are self protected during a quench by quench back from the support structure.

TABLE 3. Linear Accelerator Superconducting Solenoid Parameters

\section{Parameter}

Number of Solenoids of This Type

Beam Bore Diameter (mm)

Inner Coil Average Radius (mm)

Inner Coil Thickness (mm)

Number of Inner Coil Turns / Layers

Outer Coil Average Radius (mm)

Outer Coil Thickness (mm)

Number of Outer Coil Turns / Layers

Solenoid Average Magnetic Induction (T)

Focusing Solenoid Magnetic Length (m)

Magnet Design Current I (A)

Peak Induction in the Inner Coil Bp $(\mathrm{T})$

Magnet Conductor Ic at $4.4 \mathrm{~K}$ and $\mathrm{Bp}(\mathrm{A})$

Solenoid Stored Energy at I (MJ)
Short Magnet

$\begin{array}{cc}20 & 19 \\ 460 & 300 \\ 254 & 182 \\ 10.4 & 31.2 \\ 4840 / 8 & 21816 / 24 \\ 520.6 & 453.6 \\ 2.6 & 5.2 \\ 576 / 2 & 3512 / 4 \\ 2.1 & 4.2 \\ \sim 1.0 & \sim 1.5 \\ 469.6 & 274.0 \\ \sim 2.9 & \sim 5.8 \\ \sim 1100 & \sim 590 \\ 0.421 & 1.306\end{array}$

\section{MAGNETS AND RF CAVITIES IN THE RECIRCULATING ACCELERATOR}

Superconducting dipoles and quadrupoles were assumed for the circulating linacs for the neutrino factory[3,10,11]. The circulating linacs are designed to accelerate the muon from $2.35 \mathrm{GeV}$ to $20 \mathrm{GeV}$ in a racetrack shaped ring with four bending arcs at one end and three bending arcs at the other end. Acceleration using 201.25 MHz superconducting RF cavities occurs in the long straight sections of the ring. A switchyard at each end of the ring separates the muon beam in accordance to its energy into the bending arcs. An eighth bending arc bends the $20 \mathrm{GeV}$ muon beam into the muon storage ring.

The straight sections consist of 24 acceleration modules that are 15-meters long. The acceleration modules consist of four RF cavities with two cells in each cavity. The focusing section of the acceleration module consists of a quadrupole triplet. A schematic of the acceleration module is illustrated in Figure 5. The superconducting RF cavities in the RLA straight have an acceleration gradient of about 15 MV per meter, which is similar to the pre-acceleration linac cavities. The dominant heat load in all of the superconducting $\mathrm{RF}$ cavities is due to AC looses. AC loss is proportional to one over the cavity $\mathrm{Q}$ (a measure of energy stored in the RF cavity to energy lost per cycle). Reducing the operating temperature of the cavity from $4.4 \mathrm{~K}$ to $2.5 \mathrm{~K}$ increases the cavity $\mathrm{Q}$. The reduction of heat load due to increased cavity $Q$ will more than offsets the effect of the Carnot ratio due to the lower cavity temperature. The $\mathrm{Q}$ of the RF cavity is also a function of the acceleration gradient. 


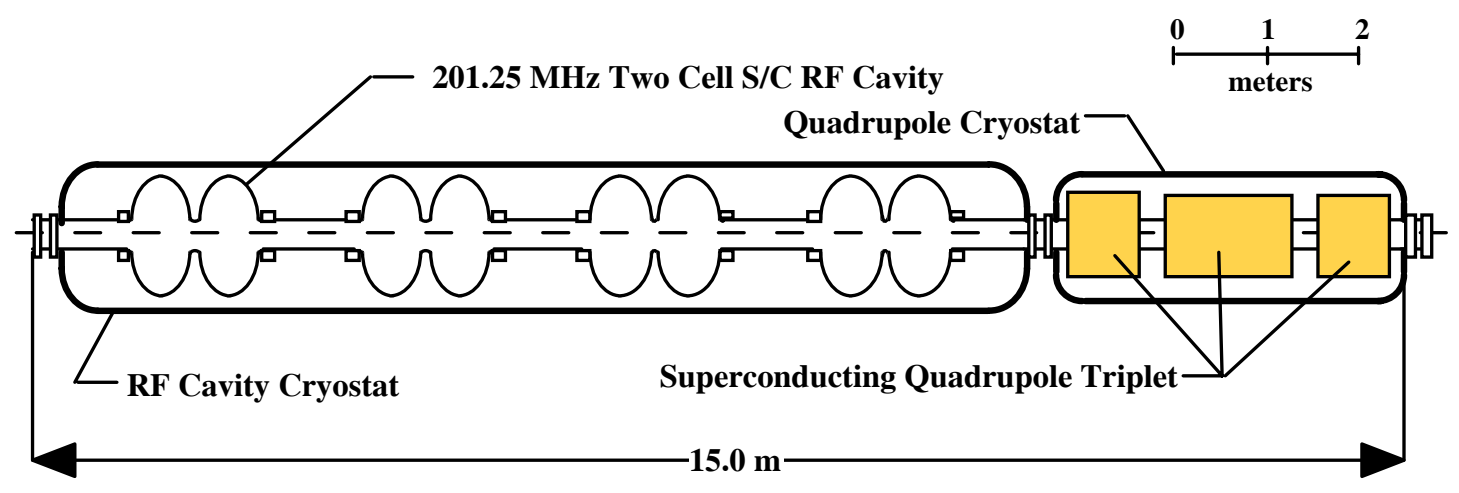

FIGURE 5. A Schematic of an Acceleration Module used in the Re-circulating Linac Straight Section. 
The stray field from the quadrupoles shown in Figure 5 is much less than the stray field from a solenoid. In order to eliminate the stray field in the RF cavities caused by the focusing quadrupole triplet, one can put a an iron shield on the quadrupole cryostat and a separate iron shield near the end of the RF cavities within the cavity cryostat.

The most recent study of the re-circulating accelerator section has proposed using superconducting quadrupoles and dipoles in entire machine. Many of these magnets can be conventional water-cooled room temperature magnets. Depending on the location of the magnet, the magnet bore diameter can vary from $100 \mathrm{~mm}$ to about $270 \mathrm{~mm}$. The proposed dipoles are picture frame dipoles. Two types of quadrupoles have been proposed; irondominated quadrupoles (with shaped iron poles and superconducting coils) and low field cosine two theta quadrupoles. The final configuration of a $20 \mathrm{GeV}$ re-circulating accelerator for the neutrino factory depends on its cost. In any event, the RF cavities in the straight sections will be superconducting with superconducting quadrupoles between the cavity modules to do the focusing. Re-circulating accelerators that accelerate muons to energies above $40 \mathrm{GeV}$ will most certainly have superconducting arc magnets.

\section{MAGNETS FOR THE NEUTRINO FACTORY STORAGE RING}

The muon storage ring for the neutrino factory is a racetrack shaped ring and is tilted so that one straight leg of the racetrack points to a neutrino detector that is 1500 to 7000 $\mathrm{km}$ from the storage ring. The muons stored in the ring decay to an electron (or positron for a positive muon) and two neutrinos. The storage ring must be designed to maximize the number of neutrinos that can reach the detector. This means that the arc length compared to the straight section length must be as short as possible. This also means that the bending field in the arc must be high and the straight section should be as long as the site will allow. One can reasonably expect to aim 35 to 40 percent of the neutrinos produced in the storage ring toward the detector. (If the storage ring arc length was zero, 50 percent of the neutrinos produced by the ring would be aimed toward the detector. A cross-section of the proposed dipole and skew quadrupole magnets for the Brookhaven National Laboratory muon storage ring is shown in Figure 6[12]. 


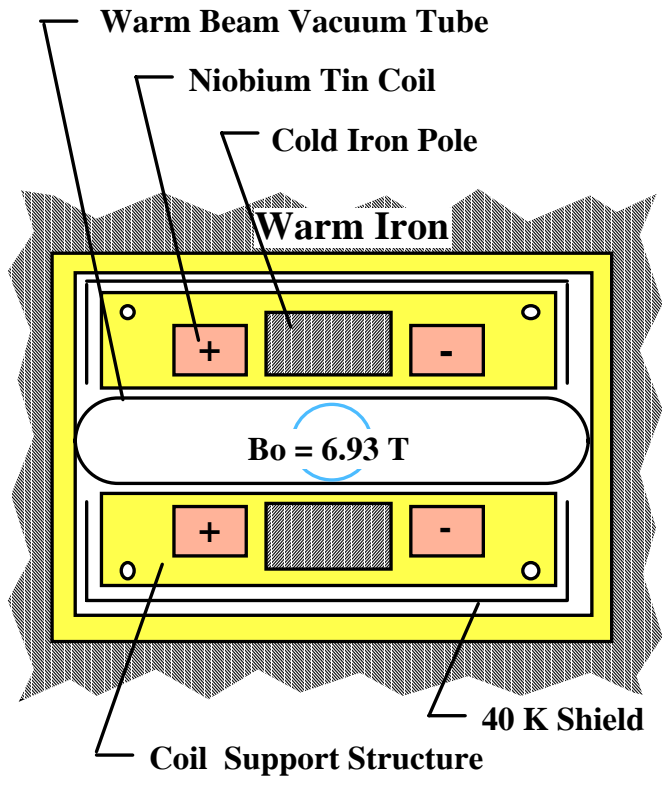

Normal Dipole

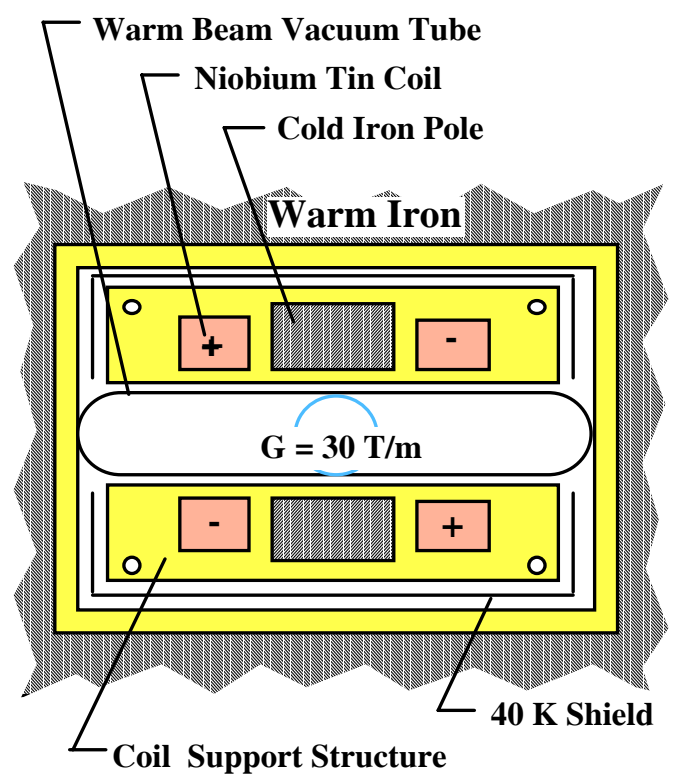

Skew Quadrupole

FIGURE 6. A Schematic of a Normal Dipole and a Skew Quadrupole for the Muon Storage Ring. The niobium tin coils and are wound as flat coils. The electron and synchrotron radiation produced by the muon decays passes between the coils to the warm iron. Only a fraction of the muon decay power ends up in the 4.4 K region. A magnet of this type appears to has an acceptable integrated field uniformity.

A lattice that has a 53-meter long arc section at each end of the racetrack involves combining open mid plane dipoles with skew quadrupoles. The dipole has the top and bottom coils carrying current at the same polarity. The skew quadrupole has coils that carry current at opposite polarity. Magnets of this type can potentially result in a dense bending arc for the storage ring, but there are some extra costs to be dealt with.

The magnets shown in Figure 6 have a peak field in the winding of about 11 T. As a result niobium-tin coils must be used for a magnet that generates less than $7 \mathrm{~T}$ on the beam axis. In order to achieve the required field uniformity standards and keep the decay electrons out of the coils, the coils have to be larger than they would be for a more conventional magnet solution. If the arc length can be made longer, a conventional separated to function arc with conventional bending magnets and quadrupoles can be used. Tungsten shields (about 30-mm thick) are used to absorb the muon decay electrons. For a $7 \mathrm{~T}$ bending field, the dipoles can be made using a niobium titanium-conductor operating at $4.4 \mathrm{~K}$. The type of arc magnets used in the neutrino factory storage ring may be site specific.

\section{CRYOGENIC HEAT LOADS FOR THE NEUTRINO FACTORY}

The cryogenic heat loads for the neutrino factory are shown in Table 4. The study done for Brookhaven assumed that the present AGS accelerator had to be upgraded using superconducting proton linacs as a pre-accelerator for the existing $30 \mathrm{GeV}$ AGS proton ring. The refrigeration for the pre-accelerator is included in Table 4. All of the superconducting RF cavities are assumed to operate at $2.5 \mathrm{~K}$. The superconducting magnets operate at $4.4 \mathrm{~K}$ except for the cable in conduit first coils of the capture solenoid that operate at $2.5 \mathrm{~K}$. The liquid hydrogen absorbers for the mini-cooler and the muon cooling sections use helium entering the absorber at $16 \mathrm{~K}$. The nominal absorber temperature is 18 to $20 \mathrm{~K}$. Magnet shields in the phase rotation, bunching and cooling sections operate at $16 \mathrm{~K}$ on up; all other magnet shields operate at $40 \mathrm{~K}$ on up. 
Table 4 shows a breakdown of the refrigeration loads for the neutrino factory. The machine is assumed to have eleven cold boxes that develop $9.2 \mathrm{~kW}$ of equivalent cooling at $4.4 \mathrm{~K}$. Ten of the cold boxes are designed to produce refrigeration $2.5 \mathrm{~K}, 4.4 \mathrm{~K}$, and $40 \mathrm{~K}$ simultaneously. The eleventh cold box must produce refrigeration at $4.4 \mathrm{~K}$ and $16 \mathrm{~K}$. Four of the $2.5 \mathrm{~K}$ cold boxes are used for the proton linacs; one $2.5 \mathrm{~K}$ cold box cools the capture solenoid system, and five of the $2.5 \mathrm{~K}$ cold boxes provide cooling for the pre-accelerator and the re-circulating linacs. The single cold box that provides cooling at $16 \mathrm{~K}$ is used to cool the phase rotation system, the mini-cooler, the muon bunchers, and the muon cooling system.

TABLE 4. Refrigeration Required for Various Neutrino Factory Components at Various Temperatures

$$
\begin{aligned}
& \text { Load Temperature (K) } \\
& \text { Machine Component }
\end{aligned}
$$

S/C Proton Linacs

Capture Solenoid System

Induction Linac I

Mini-cooler System

Induction Linacs II and III

Bunching and Muon Cooling

Linear Pre-accelerator

Re-circulating Linacs

Muon Storage Ring

Total Refrigeration

Equivalent Refrigeration at $4.4 \mathrm{~K}$

Total Equivalent Refrigeration at $4.4 \mathrm{~K}$

Installed 4.4 K Refrigeration Capacity

Input Power to the Helium Refrigerators
$2.5 \mathrm{~K}$

7.10

1.90

$--$

$--$

$--$

$---$

2.76

4.69

$---$

16.45

29.14
$4.4 \mathrm{~K}$ Refrigeration Load $(\mathrm{kW})$

\section{CONCLUDING COMMENTS}

The feasibility of a neutrino factory requires that superconducting magnets, liquid hydrogen energy absorbers and superconducting RF cavities be used. Pions must be produced on a proton target. These pions must be captured in a high magnetic field, much of which comes from a superconducting magnet. Superconducting solenoids guide the pions as they decay to muons. The muons must be cooled in a superconducting solenoid channel before they can be accelerated. The acceleration process must occur before the muons decay. The use of a superconducting acceleration system reduces the length of the accelerator section and the power used to accelerate the muons. The storage ring that produces the neutrino beam must use superconducting bending arcs, in order to maximize neutrino production. Even the neutrino detectors that are thousands of kilometers from the neutrino factory may employ cryogenics within them. Compared to a large proton storage ring, such as the LHC at CERN, the neutrino factory is small, but the applications of cryogenics and superconductors will be challenge.

\section{ACKNOWLEDGEMENTS}

The work represented in this paper was done by a large number of people, many of whom are not authors. The authors wish to acknowledge the work of the whole muon 
collider collaboration that led to the neutrino factory design. The authors specifically acknowledge the contributions of editors S.Ozaki, R. Palmer, M. Zisman, and J. Gallardo, as the editors of the Study II Feasibility report.

This work was performed at the Lawrence Berkeley National Laboratory with the support of the Office of Science, United States Department of Energy under DOE contract DE-AC03-76SF00098.

\section{REFERENCES}

1. N. Holtkamp and D. Finley eds., "A Feasibility Study of a Neutrino Source Based on a Muon Storage Ring," Fermilab-Pub-00/108E, (2000)

2. S. Ozaki, R. B. Palmer, M. S. Zisman and J. C. Gallardo eds., "Feasibility Study II of a Muon-Based Neutrino Source," BNL-52623, June 2001

3. M. S. Zisman, "Status of Neutrino Factory and Muon Collider R\&D," to be published in the 2001 Particle Accelerator Conference Proceedings, Chicago IL USA, 18-22 June 2001

4. R. J. Weggel, C. E. Pearson, and B. J. King, "Design Study for 20 T, 15 cm Bore Hybrid Magnet with Radiation-Resistant Insert for Pion Capture," to be published in the 2001 Particle Accelerator Conference Proceedings, Chicago IL USA, 18-22 June 2001

5. R. Benjegerdes, P. Bish, D. Byford, et al, "Fabrication and Test Results of a High Field Niobium Tin Superconducting Racetrack Dipole Magnet," to be published in the 2001 Particle Accelerator Conference Proceedings, Chicago IL USA, 18-22 June 2001

6. "A Thin Superconducting Solenoid for Use in a Phase Rotation Induction Linac," co-authored with J. Fockler, R. E. Lafever, D. L Vanecek, and S. S. Yu, IEEE Transactions on Applied Superconductivity $11, \mathrm{p} 2180,(2001)$

7. M. A. Green and S. Yu, "Superconducting Magnets for Induction Linac Phase-Rotation in a Neutrino Factory," submitted to Advances in Cryogenic Engineering 47 (This Proceedings) (2001)

8. M. A. Green, Y Eyssa, S. Kenny, J R. Miller, et al, "Superconducting Solenoids for the Muon Collider." co-authored with IEEE Transactions on Applied Superconductivity 10, No.1, p 196, (2000)

9. M. A. Green, J. R. Miller and S. Prestemeon, ."Superconducting Solenoids for Muon Cooling in the Neutrino Factory," submitted to Advances in Cryogenic Engineering 47 (This Proceedings) (2001)

10. H. Padamsee, "Superconducting RF - New Directions," to be published in the 2001 Particle Accelerator Conference Proceedings, Chicago IL USA, 18-22 June 2001

11. J. Dayayen, D. Douglas, L. Harwood, et al, "Issues in Acceleration of a Muon Beam for a Neutrino Factory," to be published in the 2001 Particle Accelerator Conference Proceedings, Chicago IL USA, 18-22 June 2001

12. B. Parker, M. Anerella, J. Escallier, et al, "Magnets for a Muon Storage Ring," to be published in the 2001 Particle Accelerator Conference Proceedings, Chicago IL USA, 18-22 June 2001 


\section{The Role of Superconductivity and Cryogenics In the Neutrino Factory}

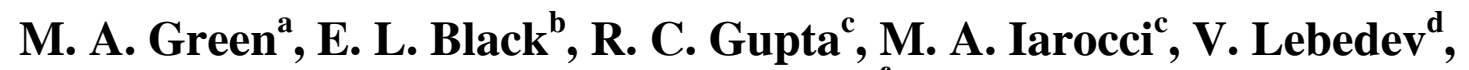
J. R. Miller ${ }^{\mathrm{e}}$, R. B. Palmer ${ }^{\mathrm{c}}$, H. S. Padamsee ${ }^{\mathrm{f}}$, B. L Parker ${ }^{\mathrm{c}}$, S. Prestemon ${ }^{\mathrm{a}}$, and R. J. Weggel ${ }^{\mathrm{c}}$

a. Lawrence Berkeley National Laboratory, Berkeley CA 94720, USA

b. Fermi National Laboratory, Batavia IL 60510, USA

c. Brookhaven National Laboratory, Upton NY 11973, USA

d. Jefferson Lab, Newport News VA 23606, USA

e. National High Magnetic Field Laboratory, Tallahassee FL 32310, USA

f. Cornell University, Ithica NY 14853, USA

July 2001

Presented at the 2001 Cryogenic Engineering Conference

Madison Wisconsin, USA

17 July through 20 July 2001 
* This work was performed at the Lawrence Berkeley National Laboratory with the support of the Office of Science, United States Department of Energy under DOE contract number DE-AC03-76SF00098. 